\title{
THE INFLUENCE OF ENVIRONMENT ON THE CHEMICAL EVOLUTION IN LOW-MASS GALAXIES
}

\author{
Yiging Liu ${ }^{1,2}$, Luis C. Ho ${ }^{2,1}$, AND ERIC Peng ${ }^{1,2}$ \\ ${ }^{1}$ Department of Astronomy, Peking University, Beijing 100871, China; yiqing.liu@pku.edu.cn \\ ${ }^{2}$ Kavli Institute for Astronomy and Astrophysics, Peking University, Beijing 100871, China \\ Received 2016 June 30; revised 2016 September 7; accepted 2016 September 7; published 2016 September 26
}

\begin{abstract}
The mean alpha-to-iron abundance ratio $([\alpha / \mathrm{Fe}])$ of galaxies is sensitive to the chemical evolution processes at early time, and it is an indicator of star formation timescale $\left(\tau_{\mathrm{SF}}\right)$. Although the physical reason remains ambiguous, there is a tight relation between $[\alpha / \mathrm{Fe}]$ and stellar velocity dispersion $(\sigma)$ among massive early-type galaxies (ETGs). However, no work has shown convincing results as to how this relation behaves at low masses. We assemble 15 data sets from the literature and build a large sample that includes 192 nearby low-mass $\left(18<\sigma<80 \mathrm{~km} \mathrm{~s}^{-1}\right)$ ETGs. We find that the $[\alpha / \mathrm{Fe}]-\sigma$ relation generally holds for low-mass ETGs, except in extreme environments. Specifically, in normal galaxy cluster environments, the $[\alpha / \mathrm{Fe}]-\sigma$ relation and its intrinsic scatter are, within uncertainties, similar for low-mass and high-mass ETGs. However, in the most massive relaxed galaxy cluster in our sample, the zero point of the relation is higher and the intrinsic scatter is significantly larger. By contrast, in galaxy groups the zero point of the relation offsets in the opposite direction, again with substantial intrinsic scatter. The elevated $[\alpha / \mathrm{Fe}]$ of low-mass ETGs in the densest environments suggests that their star formation was quenched earlier. For the low-mass ETGs in the lowest-density environments, we suggest that their more extended star formation histories suppressed their average $[\alpha / \mathrm{Fe}]$. The large scatter in $[\alpha / \mathrm{Fe}] \mathrm{may}$ reflect stochasticity in the chemical evolution of low-mass galaxies.
\end{abstract}

Key words: galaxies: abundances - galaxies: dwarf - galaxies: evolution - galaxies: formation

\section{INTRODUCTION}

Most early-type galaxies (ETGs) stopped forming stars long ago. They provide a fossil record of the star formation histories (SFHs), quenching scenarios, and mass assembly in the early universe. A powerful tracer of these early processes is the alpha-to-iron abundance ratio $([\alpha / \mathrm{Fe}])$, which is an indicator of star formation timescale $\left(\tau_{\mathrm{SF}}\right)$. In the first $\sim 0.1 \mathrm{Gyr}$ after star formation begins, enrichment of the interstellar medium is dominated by Type II supernovae (SNe II), which return ejecta with a relatively high $[\alpha / \mathrm{Fe}]$ to the interstellar medium. Afterward, Type Ia supernovae (SNe Ia) begin to contribute Ferich ejecta, and the $[\alpha / \mathrm{Fe}]$ of the entire stellar system quickly decreases as stars continue to form from this Fe-enriched gas. Over time, the system reaches an equilibrium value close to the the solar value, $[\alpha / \mathrm{Fe}] \approx 0$ (Haywood et al. 2013). Galaxies with shorter $\tau_{\mathrm{SF}}$ should reach higher $[\alpha / \mathrm{Fe}] ;[\alpha / \mathrm{Fe}]$ is sensitive to $\tau_{\mathrm{SF}}$ when $\tau_{\mathrm{SF}}$ is relatively short.

Massive ETGs $\left(\sigma \gtrsim 80 \mathrm{~km} \mathrm{~s}^{-1}\right)$ obey a tight empirical relation between $[\alpha / \mathrm{Fe}]$ and central stellar velocity dispersion $(\sigma)$, such that galaxies with larger $\sigma$ have higher $[\alpha / \mathrm{Fe}]$. The slope and zero point of the $[\alpha / \mathrm{Fe}]-\sigma$ relation do not vary much with environment (e.g., Thomas et al. 2005; McDermid et al. 2015). This relation indicates that more massive ETGs have shorter $\tau_{\mathrm{SF}}$ and, on average, quenched earlier. One possible explanation is feedback from active galactic nuclei (AGNs), as more massive ETGs host more massive central black holes (Kormendy \& Ho 2013). The expected strong quenching by AGN feedback processes (e.g., King 2003) can affect the $[\alpha / \mathrm{Fe}]-\sigma$ relation, according to the cosmological simulations of Segers et al. (2016).

Alternatively, the variation of the initial stellar mass function (IMF), fraction of SN Ia binaries, the delay time distribution of SN Ia, and stellar yields can also account for the positive correlation between $\sigma$ and $[\alpha / \mathrm{Fe}]$ (e.g., Arrigoni et al. 2010; Yates et al. 2013). However, the effects of these parameters on the $[\alpha / \mathrm{Fe}]-\sigma$ relation are highly degenerate, and there is no consensus as to which dominates. It is also possible that some massive ETGs are remnants of wet major mergers, and their high $[\alpha / \mathrm{Fe}]$ was set by the most recent starburst. However, the frequency of major mergers is not high (e.g., Lotz et al. 2011), and this scenario probably cannot fully account for the $[\alpha / \mathrm{Fe}]-\sigma$ relation.

If quenching processes determine the $[\alpha / \mathrm{Fe}]-\sigma$ relation of massive ETGs, then how this relation behaves at low mass is interesting. While the quenching mechanisms of massive galaxies are mass-dependent, low-mass galaxies are mostly quenched by environmental processes (Peng et al. 2010), such as the ram pressure stripping (Gunn \& Gott 1972), harassment (Moore et al. 1996), tidal stirring (Mayer et al. 2001), and starvation (Peng et al. 2015). Even if low-mass galaxies are not quenched by environment, feedback from SN explosions and stellar winds are still distinct from the quenching mechanisms of massive galaxies (e.g., Hopkins et al. 2011; Forbes et al. 2016).

Besides quenching, the chemical evolution histories of galaxies also depend on their mass. Peng \& Maiolino (2014) pointed out that for star-forming galaxies, the key parameter that controls galactic evolution is the timescale required to reach equilibrium. While most massive galaxies reach equilibrium in their lifetime, low-mass $\left(M_{*} \lesssim 10^{9} M_{\odot}\right)$, gasrich galaxies may not within a Hubble time. As a consequence of their non-equilibrium states, stochastic processes in lowmass galaxies, including inflow, outflow, and star formation, may be imprinted in their stellar population when they are quenched. For instance, Lee et al. (2009) found a systematically lower $\mathrm{H} \alpha / \mathrm{FUV}$ flux ratio with declining luminosity among low-mass, star-forming galaxies, which might be due to the stochastic appearance of high-mass stars at low star formation rates (SFRs; e.g., Fumagalli et al. 2011).

Therefore, it is important to investigate the $[\alpha / \mathrm{Fe}]-\sigma$ relation of low-mass ETGs. Unfortunately, no work has studied it using 
a sample of significant size, possibly due to their low surface brightness. Previous studies all had relatively large uncertainties. Sansom \& Northeast (2008), Smith et al. (2009), and Annibali et al. (2011) obtained different slopes for this relation. Using a sample of low-mass ETGs from the Virgo Cluster, Liu et al. (2016) found an $[\alpha / \mathrm{Fe}]-\sigma$ relation with larger scatter and, based on a correlation between $[\alpha / \mathrm{Fe}]$ and distance from the cluster center, concluded that low-mass ETGs are more governed by environment instead of mass. However, Vargas et al. (2014), from a sample of Local Group dwarf spheroidal galaxies (dSphs), claimed a large scatter without any environmental dependence.

To study the early baryonic processes in low-mass galaxies, we collect literature data for a relatively large sample to systematically study the $[\alpha / \mathrm{Fe}]-\sigma$ relation of low-mass ETGs. We are interested in not only the form of the relation, but also its intrinsic scatter, which provides information about the early SFHs of low-mass galaxies.

\section{DATA}

We assemble from the literature a sample of 708 ETGs, which spans a wide range in mass and environment, with measurements of both $[\alpha / \mathrm{Fe}]$ and $\sigma$. In order to make the sample as homogeneous as possible and allow for intercomparison, we only collect $[\alpha / \mathrm{Fe}]$ measurements derived from single stellar population models, using Lick index measurements of integrated-light spectra. As a result, galaxies as faint as the Local Group $\mathrm{dSphs}$ are excluded from our sample, and no measurement is from high redshift. Our final sample has $\sigma$ ranging from 18 to $360 \mathrm{~km} \mathrm{~s}^{-1}$, among them 192 low-mass ETGs with $\sigma<80 \mathrm{~km} \mathrm{~s}^{-1}$.

The $[\alpha / \mathrm{Fe}]$ values of most of the data sets used in our analysis were derived following the methodology of Thomas et al. (2003) or Schiavon (2007). To eliminate the systematic offsets between these two models, we apply the following correction from Smith et al. (2009) to adjust the values of Schiavon (2007; Sch) to the scale of Thomas et al. (2003; TMB):

$$
[\alpha / \mathrm{Fe}]^{\mathrm{TMB}}=0.99[\mathrm{Mg} / \mathrm{Fe}]^{\mathrm{Sch}}+0.06[\mathrm{Fe} / \mathrm{H}]^{\mathrm{Sch}}+0.02 .
$$

The models of Proctor \& Sansom (2002) and Sansom \& Northeast (2008) are the same as that of Thomas et al. (2003), except that they did not account for the $[\alpha / \mathrm{Fe}]$ bias in the solar neighborhood; this, however, has negligible effects on our results. The models of Annibali et al. (2007, 2011) are consistent with those of Thomas et al. (2003). A more detailed discussion is given in Section 3.3.

Our data sets were acquired using a range of aperture sizes, although most were between $1 / 8$ to 1 galaxy effective radius $\left(R_{\mathrm{e}}\right)$. The more massive galaxies were measured from more central regions, whereas low-mass objects tend to cover a relatively larger area. We note, however, that ETGs generally have very weak radial gradients in $[\alpha / \mathrm{Fe}]$ (e.g., Spolaor et al. 2010), and thus any systematic offsets in $[\alpha / \mathrm{Fe}]$ from different apertures should be small. As for velocity dispersions, Cappellari et al. $(2013 \mathrm{a}, 2013 \mathrm{~b})$ provide $\sigma$ measurements at both $R_{\mathrm{e}} / 8$ and $R_{\mathrm{e}}$ of 260 massive ETGs. The mean absolute offset between these two apertures is 0.05 dex. As illustrated in Figure 1 (yellow line in the bottom right corner), this level of potential systematic offset cannot strongly affect the global $[\alpha / \mathrm{Fe}]-\sigma$ relation.

\section{RESULTS}

\subsection{The $[\alpha / \mathrm{Fe}]-\sigma$ Relation}

Figure 1 displays the $[\alpha / \mathrm{Fe}]-\sigma$ diagram of ETGs across a wide mass range, using the entire sample we collected. Different subsamples are plotted in different colors and symbols. Whereas the upper end of the distribution clearly obeys the wellknown tight correlation, we see that the scatter notably increases toward the lower end. The transition occurs at $\log \sigma \approx 1.9$ $\left(\sigma=80 \mathrm{~km} \mathrm{~s}^{-1}\right)$, which we use as the dividing line between the high- $\sigma$ (high-mass) and low- $\sigma$ (low-mass) groups.

We begin by characterizing the $[\alpha / \mathrm{Fe}]-\sigma$ relation for the high- $\sigma$ group. To fit the linear relation and calculate their intrinsic scatter, we use MPFITEXY, which is the FITEXY estimator of Press et al. (1992), as modified by Tremaine et al. (2002). We allow for asymmetric errors. To estimate the uncertainties caused by the potentially biased sampling, we perform 5000 bootstrap iterations on each fit to calculate the errors of our fitting parameters. The results, listed in Table 1 and illustrated in Figure 1 (yellow dashed line and band), agree well with previous work (e.g., Thomas et al. 2005; McDermid et al. 2015). The intrinsic scatter of the $[\alpha / \mathrm{Fe}]-\sigma$ relation for the high- $\sigma$ group is very small, only $\epsilon_{0}=(0.064 \pm 0.004)$ dex.

The large measurement uncertainties for the low-mass ETGs prevent us from performing an unconstrained fit for these objects. To first obtain a qualitative assessment, we divide the low-mass group into three bins of $\log \sigma: 1.2-1.5,1.5-1.7$, and 1.7-1.9. In Figure 1, the green stars show the weighted average values of these bins. The two bins with higher $\sigma$ basically follow the extrapolation of the relation of the high-mass group. Fixing the slope to that of the high-mass end $(a=0.280)$, we find a statistically similar zero point but an intrinsic scatter $\left[\epsilon_{0}=(0.126 \pm 0.014)\right.$ dex] two times larger than that of the high-mass objects (Table 1; orange dashed line and band in Figure 1).

\subsection{The Environmental Dependence of the $[\alpha / \mathrm{Fe}]-\sigma$ Relation}

The large intrinsic scatter of the low-mass objects, however, is not contributed by the entire sample. Most of the objects with the highest values of $[\alpha / \mathrm{Fe}]$ are from the study of Chilingarian et al. (2008), which targeted the galaxy cluster Abell 496, the densest environment in our compilation. At the same time, the galaxies with the lowest $[\alpha / \mathrm{Fe}]$ are from low-density, poor groups. This motivates us to further test the role of environment in driving the large scatter of the $[\alpha / \mathrm{Fe}]-\sigma$ relation in the lowmass regime. We divide our low-mass sample into three bins of environmental density and fit their relations, as before, with fixed slope (Table 1): high (Abell 496), moderate (Coma, Virgo, and Fornax Clusters, as well as the Shapley Supercluster), and low (groups).

Figure 2 displays the residuals of the $[\alpha / \mathrm{Fe}]-\sigma$ relation, $\delta[\alpha / \mathrm{Fe}]$, as a function of $\sigma$, for all low-mass galaxies with reliable environment information. The red, green, and blue triangles represent the weighted-mean ${ }^{3}[\alpha / \mathrm{Fe}]$ and $\sigma$ of the subsamples residing in environments of high, moderate, and low density, respectively; their corresponding zero points and intrinsic scatter are given by the dashed lines and colored bands.

\footnotetext{
3 Because some references did not publish errors on $\sigma$, the mean $\sigma$ is not weighted.
} 


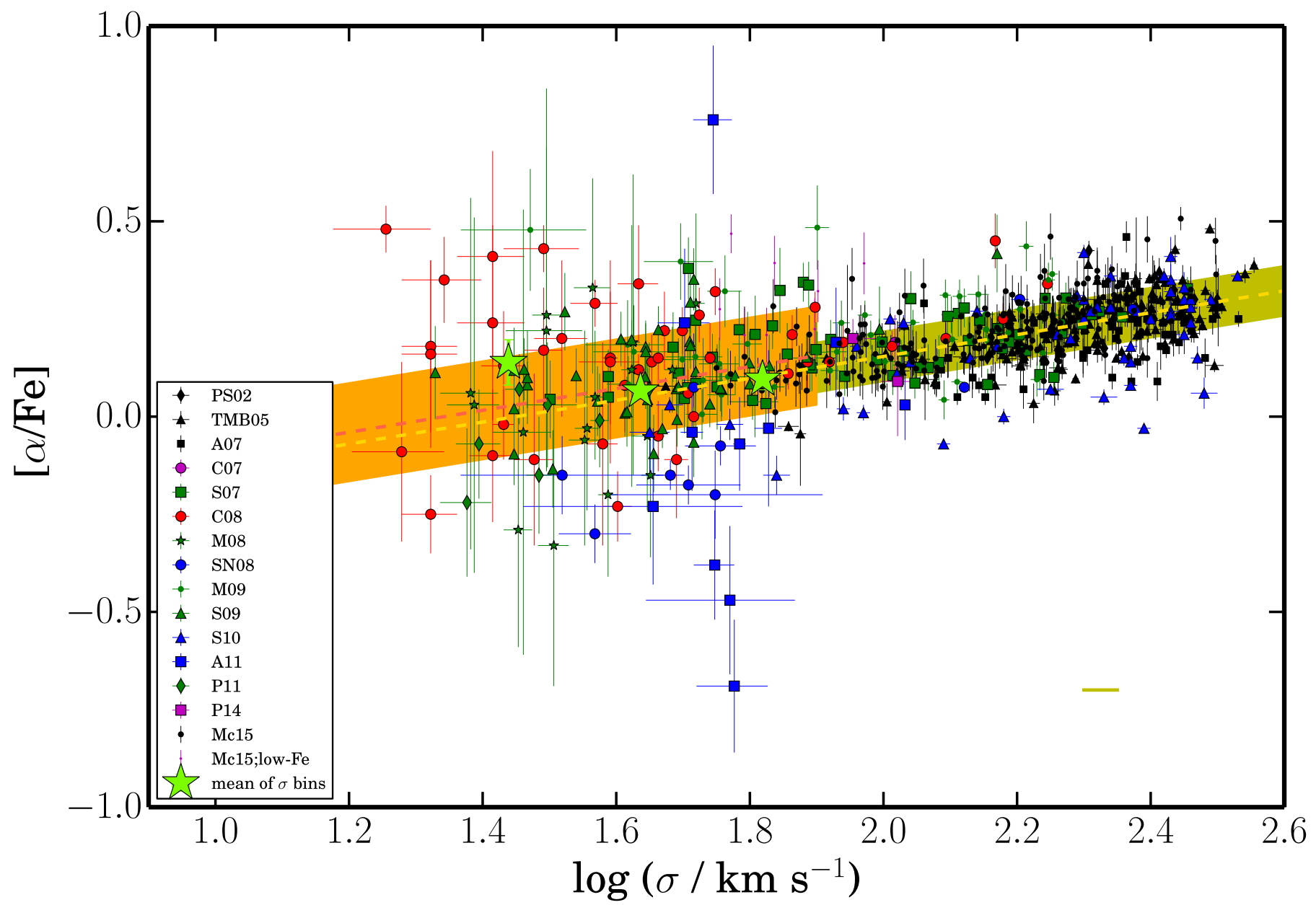

Figure 1. Relation between $[\alpha / \mathrm{Fe}]$ derived from single stellar population models and $\sigma$ of ETGs. Different colors and symbols represent different subsamples, whose references are listed in the legend: Proctor \& Sansom (2002; PS02), Thomas et al. (2005; TMB05), Annibali et al. (2007; A07), Chilingarian et al. (2007; C07), Smith et al. (2007; S07), Chilingarian et al. (2008; C08), Michielsen et al. (2008; M08), Sansom \& Northeast (2008; SN08), Matković et al. (2009; M09), Smith et al. (2009; S09), Spolaor et al. (2010; S10), Annibali et al. (2011; A11), Paudel et al. (2011; P11), Paudel et al. (2014; P14), and McDermid et al. (2015; Mc15). The $\sigma$ measurements of the Michielsen et al. (2008) and Paudel et al. (2011) sources are from Toloba et al. (2014). The galaxies in our sample are divided into two groups by $\log \sigma=1.9$. The green stars in the low-mass range show the weighted mean of three bins of $\sigma$, which are $\log \sigma=1.2-1.5,1.5-1.7$, and 1.7-1.9. The yellow dashed line and band display the relation and intrinsic scatter fitted by the high- $\sigma$ group. In the low- $\sigma$ range, they are displayed by the orange dashed line and band, and the slope is fixed to be the same as that of the high- $\sigma$ group in the fitting. The potential offset of $\sigma$ between different subsamples is shown at the bottom right corner; this has negligible effects on our results.

Table 1

The $[\alpha / \mathrm{Fe}]-\sigma$ Relation for Different Subsamples

\begin{tabular}{lccc}
\hline \hline Group & $a$ & $b$ & $\epsilon_{0}$ \\
\hline High- $\sigma$ & $0.280 \pm 0.023$ & $-0.406 \pm 0.051$ & $0.064 \pm 0.004$ \\
\hline Low- $\sigma$ & & & \\
\hline Total & $0.280^{\mathrm{a}}$ & $-0.376 \pm 0.011$ & $0.126 \pm 0.014$ \\
H-Env & $0.280^{\mathrm{a}}$ & $-0.311 \pm 0.030$ & $0.126 \pm 0.032$ \\
M-Env & $0.280^{\mathrm{a}}$ & $-0.364 \pm 0.012$ & $0.058 \pm 0.015$ \\
L-Env & $0.280^{\mathrm{a}}$ & $-0.661 \pm 0.051$ & $0.145 \pm 0.064$ \\
\hline
\end{tabular}

Note. $[\alpha / \mathrm{Fe}]=a \log \sigma+b$. The intrinsic scatter is $\epsilon_{0} . \mathrm{H} / \mathrm{M} / \mathrm{L}$-Env represent the groups of low-mass ETGs with different levels of environmental density.

${ }^{a}$ No error measurement, because the slope is fixed.

The majority of the low-mass ETGs in our study are from moderate-density environments. Similar to the situation for the entire sample of low-mass objects, the moderate-density subsample has a zero point and intrinsic scatter consistent with those of the massive end. This implies that the large intrinsic scatter of the low-mass sample comes mostly from objects in extreme environments. Relative to the high-mass end, low-mass objects in high-density environments have a higher zero point and larger scatter over $2 \sigma$ significance; those in low-density environments are even more notable in terms of their lower zero point $(5 \sigma$ significance) and increased scatter ( $\sigma$ significance).

\subsection{An Empirical Check}

Because $[\alpha / \mathrm{Fe}]$ is a model-dependent parameter, its values derived from different stellar population models may have systematic offsets. To mitigate these effects, we only use measurements derived from Lick indices, and we homogenize the derived $[\alpha / \mathrm{Fe}]$ values to a common system (see Section 2). Nevertheless, it would be desirable to verify whether our results are robust with respect to model uncertainties. Independent of models, $\mathrm{Mgb} /\langle\mathrm{Fe}\rangle$ is the most direct indicator of $\alpha / \mathrm{Fe}$ among the Lick indices. Although this index ratio is also dependent on age and metallicity, it is most sensitive to 


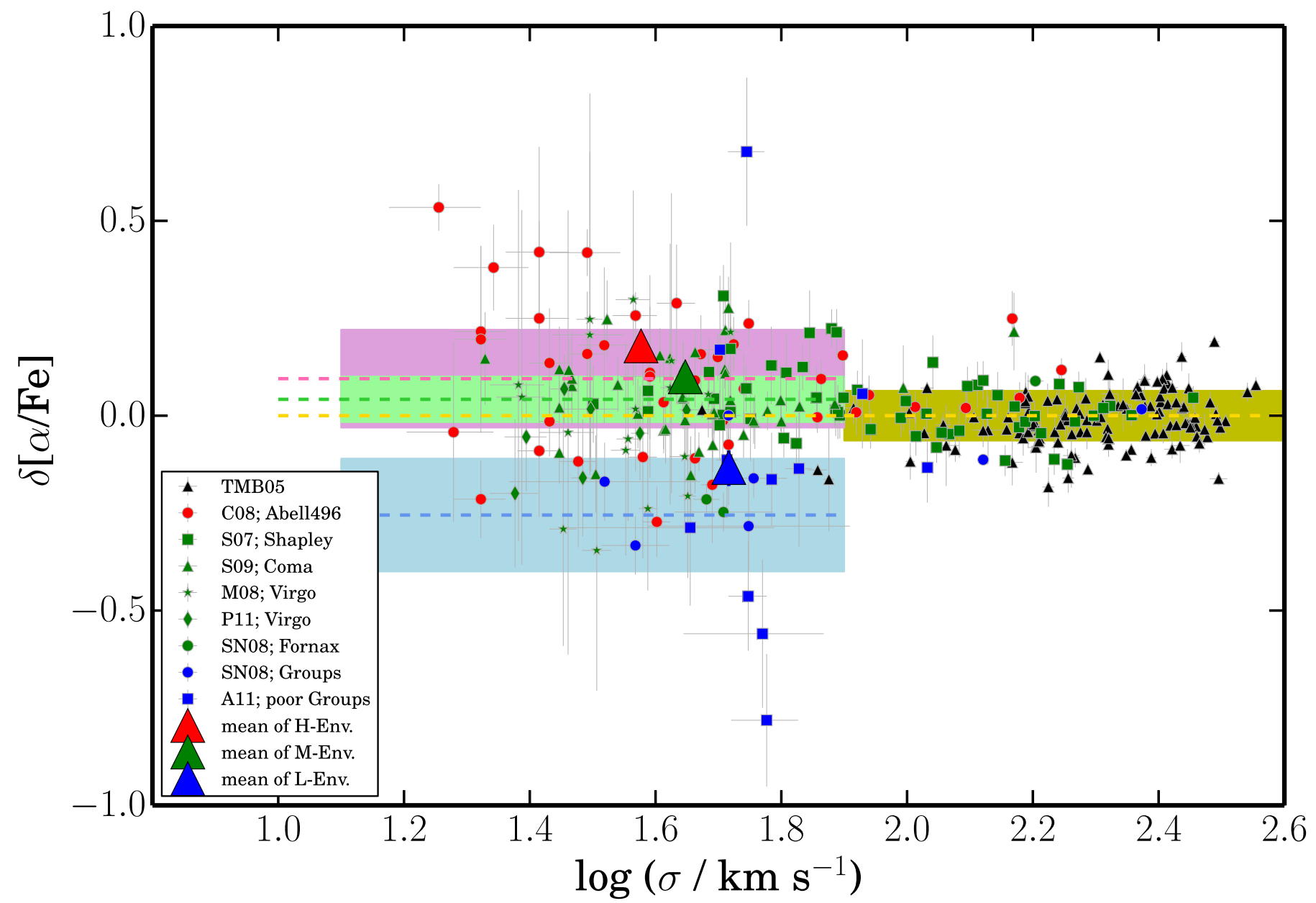

Figure 2. Distribution of $[\alpha / \mathrm{Fe}]$ residuals with $\sigma$ and environment. The residuals of $\delta[\alpha / \mathrm{Fe}]$ are calculated from the relation fitted by the high- $\sigma$ group. Except for the objects from the sample of TMB05 (black triangles), which is included for reference, only the samples that contain low- $\sigma$ galaxies and have environmental information are plotted. The samples with the highest- and lowest-density environments are color-coded red and blue, respectively; those located in intermediate-density environments are shown in green. The corresponding colored triangles represent the weighted-mean $[\alpha / \mathrm{Fe}]$ and mean $\sigma$ of the three environmental subsamples. Dashed lines give the zero-point offsets with respect to the high- $\sigma$ group (yellow dashed line), and colored bands denote their respective intrinsic scatter.

$\alpha / \mathrm{Fe}$. Seven of our literature sources published measurements of both $\mathrm{Mgb}$ and $\langle\mathrm{Fe}\rangle$, covering the full range of environmental density probed in this study. Figure 3 presents the equivalent of the $[\alpha / \mathrm{Fe}]-\sigma$ relation (Figures 1 and 2), wherein we replace $[\alpha / \mathrm{Fe}]$ with $\log (\mathrm{Mgb} /\langle\mathrm{Fe}\rangle)$. Table 2 lists the fitting parameters of the $\log (\mathrm{Mgb} /\langle\mathrm{Fe}\rangle)-\sigma$ relation for the different subsamples.

All the main conclusions from Sections 3.1 and 3.2 hold. As before, massive ETGs follow a very tight relation between $\log (\mathrm{Mgb} /\langle\mathrm{Fe}\rangle)$ and $\sigma$ with relatively small intrinsic scatter, and, within the uncertainties, this relation can be extrapolated into the low-mass regime, but with larger intrinsic scatter. When low-mass ETGs are grouped by their environments, those that reside in moderate-density clusters have a relation that is indistinguishable from that of massive ETGs in terms of zero point and intrinsic scatter, while those in the highest- and lowest-density environments have larger intrinsic scatter ( $3 \sigma$ and $1 \sigma$ significance) with higher and lower zero points ( $3 \sigma$ significance), respectively. This analysis, making use of a separate, model-independent estimate of $[\alpha / \mathrm{Fe}]$, confirms that the tight $[\alpha / \mathrm{Fe}]-\sigma$ relation for massive ETGs can be extrapolated toward low masses, but that its intrinsic scatter and zero point differ in extreme environments.

\section{DISCUSSION}

\subsection{The Origin of the $[\alpha / \mathrm{Fe}]-\sigma$ Relation}

The mean $[\alpha / \mathrm{Fe}]$ of galaxies arises from a complex interplay of many factors, including $\tau_{\mathrm{SF}}$, IMF, stellar yields, SN Ia explosion rate and delay time distribution, and gas inflow and outflow. The departure of the standard $[\alpha / \mathrm{Fe}]-\sigma$ relation toward low masses, at least for ETGs residing in high- and lowdensity environments, implies that some of the physical drivers underlying this empirical scaling relation evidently change under these circumstances. While it is difficult to assess the relative importance of the various potential factors, here we highlight the possible role of $\tau_{\mathrm{SF}}$ and $\mathrm{SFH}$.

Two often-discussed candidate quenching mechanisms for massive ETGs are AGN feedback (e.g., Croton et al. 2006) and halo quenching (e.g., Dekel \& Birnboim 2006). These quenching mechanisms appear to be mass-dependent, and more massive ETGs have shorter $\tau_{\mathrm{SF}}$. However, low-mass ETGs are quenched by different mechanisms. Some of them were satellite galaxies and were quenched by environmental processes, such as ram pressure stripping and strangulation, when they fell into the dark matter halos of nearby host galaxies (Gunn \& Gott 1972; Peng et al. 2015). Their $\tau_{\mathrm{SF}}$ might 


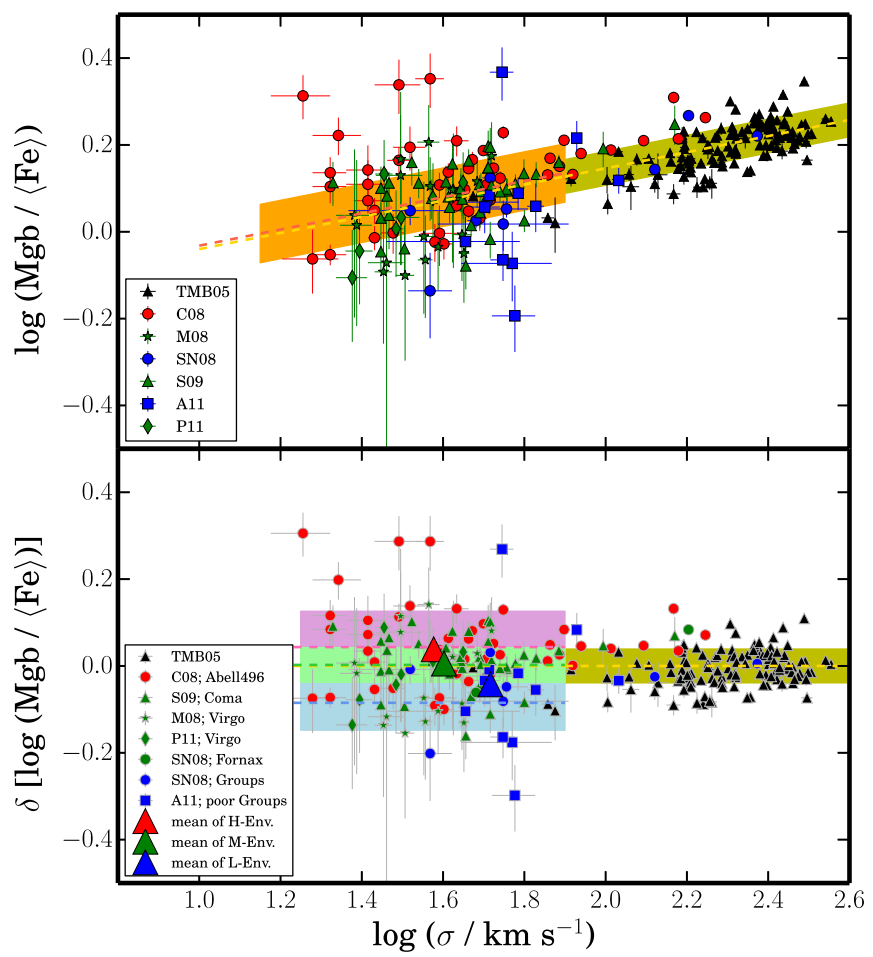

Figure 3. Upper and bottom panels are parallel plots to Figures 1 and 2, respectively, with $[\alpha / \mathrm{Fe}]$ replaced by $\log (\mathrm{Mgb} /\langle\mathrm{Fe}\rangle)$, illustrating that the relations are model-independent. Symbols, lines, and colors have the same meaning as in Figures 1 and 2.

Table 2

The $\log (\mathrm{Mgb} /\langle\mathrm{Fe}\rangle)-\sigma$ Relation for Different Subsamples

\begin{tabular}{lccc}
\hline \hline Group & \multicolumn{1}{c}{$a$} & $b$ & $\epsilon_{0}$ \\
\hline High- $\sigma$ & $0.186 \pm 0.016$ & $-0.226 \pm 0.037$ & $0.039 \pm 0.002$ \\
\hline Low- $\sigma$ & & & \\
\hline Total & $0.186^{\mathrm{a}}$ & $-0.218 \pm 0.006$ & $0.067 \pm 0.008$ \\
H-Env & $0.186^{\mathrm{a}}$ & $-0.182 \pm 0.014$ & $0.083 \pm 0.014$ \\
M-Env & $0.186^{\mathrm{a}}$ & $-0.224 \pm 0.007$ & $0.040 \pm 0.007$ \\
L-Env & $0.186^{\mathrm{a}}$ & $-0.311 \pm 0.025$ & $0.063 \pm 0.023$ \\
\hline
\end{tabular}

Note. $\log (\mathrm{Mgb} /\langle\mathrm{Fe}\rangle)=a \log \sigma+b . \epsilon_{0}$ is the intrinsic scatter. $\mathrm{H} / \mathrm{M} / \mathrm{L}-$ Env represent the groups of low-mass ETGs with different levels of environmental density.

${ }^{a}$ No error measurement, because the slope is fixed.

be more driven by environment than mass. Moreover, galaxies with shallower potential wells are expected to experience even stronger environmental effects, which may result in shorter $\tau_{\mathrm{SF}}$ and higher $[\alpha / \mathrm{Fe}]$. The effect of environment should be especially acute given that most low-mass ETG samples reside in galaxy clusters. Under these circumstances, we naively expect low-mass ETGs to exhibit an $[\alpha / \mathrm{Fe}]-\sigma$ correlation with negative slope, opposite to that actually observed. A positive $[\alpha / \mathrm{Fe}]-\sigma$ correlation also contradicts the simplest expectations from internal quenching mechanisms such as radiative and energetic feedback from $\mathrm{SNe}$ and high-mass stars, which should operate more effectively and stop star formation earlier (thus boosting $[\alpha / \mathrm{Fe}])$ in lower-mass galaxies.

The $[\alpha / \mathrm{Fe}]-\sigma$ relation may also be a consequence of SFH. From cosmological semi-analytical simulations, De Lucia et al.
(2006) showed that more massive ETGs had more peaked SFR distributions, which reached their peak at higher redshifts. However, they only studied massive galaxies with stellar masses larger than $4 \times 10^{9} M_{\odot}$. Moreover, other studies have not been able to reproduce the positive correlation between $[\alpha / \mathrm{Fe}]$ and $\sigma$ for massive ETGs without including special recipes (e.g., Arrigoni et al. 2010; Yates et al. 2013; Segers et al. 2016). Segers et al. (2016) extended their simulations to galaxies of lower masses and reproduced a positive $[\alpha / \mathrm{Fe}]-\sigma$ correlation for stellar masses between $10^{8} M_{\odot}$ and $10^{10} M_{\odot}$. It is unclear, however, whether these simulations apply to the situation at hand. Segers et al. only considered central galaxies that are still forming stars at $z \approx 0$.

\subsection{The Role of Environment}

We find that low-mass ETGs from the densest environments are, on average, offset toward larger $[\alpha / \mathrm{Fe}]$. A plausible implication of this finding is that high environmental density induces short $\tau_{\mathrm{SF}}$. As discussed in Section 4.1, low-mass galaxies in high-density regions might experience environmental quenching earlier, which would systematically elevate their $[\alpha / \mathrm{Fe}]$. At the same time, environmental density also influences early star formation processes in low-mass galaxies. Santos et al. (2015) found that star-forming galaxies in the central regions of high-redshift clusters have higher SFRs. This suggests that present-day low-mass ETGs residing in denser environments may have experienced more intense star formation at early times, resulting in their higher observed $[\alpha / \mathrm{Fe}]$. By contrast, the average $[\alpha / \mathrm{Fe}]$ of low-mass ETGs is lower in galaxy groups, indicating more extended SFHs of low-mass galaxies in low-density environments. This is consistent with the findings of Geha et al. (2012). All the isolated low-mass galaxies without $\mathrm{H} \alpha$ emission in their sample showed evidence of recent starbursts, implying bursty SFHs over an extended period.

In both of the extreme (high- and low-density) environments highlighted in this work, the large scatter of $[\alpha / \mathrm{Fe}]$ at low mass can be explained by stochastic chemical evolution processes in non-equilibrium systems. According to the main sequence of star-forming galaxies (e.g., Wuyts et al. 2011), low-mass galaxies have low SFRs. Under such conditions, massive stars and SNe II appear stochastically because the number of forming stars is not large enough to sample the IMF completely (e.g., Lee et al. 2009; Fumagalli et al. 2011). Furthermore, some $\mathrm{SNe}$ in the early universe may produce special abundance patterns, such as both low $[\alpha / \mathrm{Fe}]$ and low $[\mathrm{Fe} / \mathrm{H}]$ (Kobayashi et al. 2014; Simon et al. 2015). The dispersion in $[\alpha / \mathrm{Fe}]$ also reflects the details of the gas evolution after the appearance of the first generation of stars. For example, star formation may cease in hot bubbles after the surrounding neutral gas is ionized, but it may restart in colder regions afterward (e.g., Sobral et al. 2015). Outflows with high $[\alpha / \mathrm{Fe}]$ ejected by these stars, if still bound to the halo, might fall back and contribute to subsequent star formation. Both scenarios would temporally increase the mean galactic $[\alpha / \mathrm{Fe}]$.

Yet, it is curious that the large scatter in $[\alpha / \mathrm{Fe}]$ is not seen in all low-mass ETGs, but rather only in the subset residing in extremely high- or low-density environments. After all, the intrinsic scatter of the $[\alpha / \mathrm{Fe}]-\sigma$ relation over the same lowmass range in normal galaxy clusters is as tight as in high-mass ETGs. We have no explanation for this. 


\section{SUMMARY}

We assemble 708 nearby ETGs from 15 literature data sets to investigate the $[\alpha / \mathrm{Fe}]-\sigma$ relation across a wide range of galactic masses and environments. Our sample spans $\sigma \approx 18-360 \mathrm{~km} \mathrm{~s}^{-1}$, with environments covering both galaxy clusters and groups, among them 192 low-mass ETGs with $\sigma<80 \mathrm{~km} \mathrm{~s}^{-1}$.

We recover the well-known tight $[\alpha / \mathrm{Fe}]-\sigma$ relation among massive $\left(\sigma \gtrsim 80 \mathrm{~km} \mathrm{~s}^{-1}\right)$ ETGs. The relation extends into the low-mass regime, especially for low-mass ETGs residing in moderate-density galaxy clusters and superclusters, which maintain the same zero point and intrinsic scatter defined by high-mass systems. By contrast, the zero point of the $[\alpha / \mathrm{Fe}]-\sigma$ relation for low-mass ETGs is higher in the densest cluster and suppressed in the lower-density groups; the intrinsic scatter of the relation is strikingly larger in both of these extreme environments.

We suggest that both mass and environment regulate $[\alpha / \mathrm{Fe}]$ in low-mass ETGs. While $[\alpha / \mathrm{Fe}]$ is governed by total galactic mass in normal galaxy clusters, it is elevated by the earlier quenching in the densest environments and suppressed in due to the more extended SFHs in galaxy groups. The overall low SFR of low-mass systems induces stochasticity in their chemical enrichment history, which can plausibly account for the observed increased scatter in $[\alpha / \mathrm{Fe}]$.

We thank an anonymous referee for comments that helped improve our paper. Y.L. is grateful for the great academic environment at KIAA; she also appreciates discussions with Yingjie Peng and Andrew McWilliam, as well as the help from Minjin Kim with the fitting algorithms. This work was supported by grant 2016YFA0400702 from the Ministry of Science and Technology of China. We also acknowledge support from the National Natural Science Foundation of China under grant Nos. 11573002 and 11473002, and from the Strategic Priority Research Program, "The Emergence of Cosmological Structures," of the Chinese Academy of Sciences, under grant No. XDB09030102 and XDB09000105.

\section{REFERENCES}

Annibali, F., Bressan, A., Rampazzo, R., Zeilinger, W. W., \& Danese, L. 2007, A\&A, 463, 455

Annibali, F., Grützbauch, R., Rampazzo, R., Bressan, A., \& Zeilinger, W. W. 2011, A\&A, 528, A19

Arrigoni, M., Trager, S. C., Somerville, R. S., \& Gibson, B. K. 2010, MNRAS, 402, 173

Cappellari, M., McDermid, R. M., Alatalo, K., et al. 2013, MNRAS, 432, 1862

Cappellari, M., Scott, N., Alatalo, K., et al. 2013, MNRAS, 432, 1709
Chilingarian, I., Cayatte, V., Chemin, L., et al. 2007, A\&A, 466, L21

Chilingarian, I. V., Cayatte, V., Durret, F., et al. 2008, A\&A, 486, 85

Croton, D. J., Springel, V., White, S. D. M., et al. 2006, MNRAS, 365, 11

Dekel, A., \& Birnboim, Y. 2006, MNRAS, 368, 2

De Lucia, G., Springel, V., White, S. D. M., Croton, D., \& Kauffmann, G. 2006, MNRAS, 366, 499

Forbes, J. C., Krumholz, M. R., Goldbaum, N. J., \& Dekel, A. 2016, Natur, 535,523

Fumagalli, M., da Silva, R. L., \& Krumholz, M. R. 2011, ApJL, 741, L26

Geha, M., Blanton, M. R., Yan, R., \& Tinker, J. L. 2012, ApJ, 757, 85

Gunn, J. E., \& Gott, J. R., III 1972, ApJ, 176, 1

Haywood, M., Di Matteo, P., Lehnert, M. D., Katz, D., \& Gómez, A. 2013, A\&A, 560, A109

Hopkins, P. F., Quataert, E., \& Murray, N. 2011, MNRAS, 417, 950

King, A. 2003, ApJL, 596, L27

Kobayashi, C., Ishigaki, M. N., Tominaga, N., \& Nomoto, K. 2014, ApJL, 785, L5

Kormendy, J., \& Ho, L. C. 2013, ARA\&A, 51, 511

Lee, J. C., Gil de Paz, A., Tremonti, C., et al. 2009, ApJ, 706, 599

Liu, Y., Peng, E. W., Blakeslee, J., et al. 2016, ApJ, 818, 179

Lotz, J. M., Jonsson, P., Cox, T. J., et al. 2011, ApJ, 742, 103

Matković, A., Guzmán, R., Sánchez-Blázquez, P., et al. 2009, ApJ, 691, 1862

Mayer, L., Governato, F., Colpi, M., et al. 2001, ApJ, 559, 754

McDermid, R. M., Alatalo, K., Blitz, L., et al. 2015, MNRAS, 448, 3484

Michielsen, D., Boselli, A., Conselice, C. J., et al. 2008, MNRAS, 385, 1374

Moore, B., Katz, N., Lake, G., Dressler, A., \& Oemler, A. 1996, Natur, 379, 613

Paudel, S., Lisker, T., Hansson, K. S. A., \& Huxor, A. P. 2014, MNRAS, 443, 446

Paudel, S., Lisker, T., \& Kuntschner, H. 2011, MNRAS, 413, 1764

Peng, Y., Maiolino, R., \& Cochrane, R. 2015, Natur, 521, 192

Peng, Y.-j., Lilly, S. J., Kovač, K., et al. 2010, ApJ, 721, 193

Peng, Y.-j., \& Maiolino, R. 2014, MNRAS, 443, 3643

Press, W. H., Teukolsky, S. A., Vetterling, W. T., \& Flannery, B. P. 1992 , Numerical Recipes: The Art of Scientific Computing (Cambridge: Cambridge Univ. Press)

Proctor, R. N., \& Sansom, A. E. 2002, MNRAS, 333, 517

Sansom, A. E., \& Northeast, M. S. 2008, MNRAS, 387, 331

Santos, J. S., Altieri, B., Valtchanov, I., et al. 2015, MNRAS, 447, L65

Schiavon, R. P. 2007, ApJS, 171, 146

Segers, M. C., Schaye, J., Bower, R. G., et al. 2016, MNRAS, 461, L102

Simon, J. D., Jacobson, H. R., Frebel, A., et al. 2015, ApJ, 802, 93

Smith, R. J., Lucey, J. R., Hudson, M. J., et al. 2009, MNRAS, 392, 1265

Smith, R. J., Lucey, J. R., \& Hudson, M. J. 2007, MNRAS, 381, 1035

Sobral, D., Matthee, J., Darvish, B., et al. 2015, ApJ, 808, 139

Spolaor, M., Kobayashi, C., Forbes, D. A., Couch, W. J., \& Hau, G. K. T. 2010, MNRAS, 408, 272

Thomas, D., Maraston, C., \& Bender, R. 2003, MNRAS, 339, 897

Thomas, D., Maraston, C., Bender, R., \& Mendes de Oliveira, C. 2005, ApJ, 621,673

Toloba, E., Guhathakurta, P., Peletier, R. F., et al. 2014, ApJS, 215, 17

Tremaine, S., Gebhardt, K., Bender, R., et al. 2002, ApJ, 574, 740

Vargas, L. C., Geha, M. C., \& Tollerud, E. J. 2014, ApJ, 790, 73

Wuyts, S., Förster Schreiber, N. M., van der Wel, A., et al. 2011, ApJ, 742, 96

Yates, R. M., Henriques, B., Thomas, P. A., et al. 2013, MNRAS, 435,3500 